\title{
Sequential Targeted Temperature Management: Case Report and Literature Review
}

\author{
Sebastian Casillas $^{1}$, Joseph Varon ${ }^{2}$, Salim Surani ${ }^{3}$ \\ 1. Research, Dorrington Medical Associates, Houston, USA 2. Critical Care, University of Texas Health Science Center \\ and United General Hospital, Houston, USA 3. Internal Medicine, Texas A\&M Health Science Center, Temple, USA
}

Corresponding author: Salim Surani, srsurani@hotmail.com

\begin{abstract}
We present the case of a 59-year-old gentleman with a history of nonmetastatic tonsillar malignancy and radiation chondronecrosis, who underwent targeted temperature management (TTM) in a sequential manner. The first time the patient underwent therapeutic cooling occurred after he developed a respiratory arrest followed by a cardiac arrest and prolonged hypoxemia after a diagnostic laryngoscopy. The patient was kept at $32^{\circ} \mathrm{C}$ for 24 hours, and 48 hours after rewarming woke up neurologically intact. However, six hours post-extubation, he suffered upper airway obstruction, followed by a prolonged cardiac arrest. Return of spontaneous circulation on this second episode was achieved after 45 minutes of resuscitation maneuvers. The patient was cooled again and kept at $32^{\circ} \mathrm{C}$ for 48 hours. Five days later, the patient recovered, with an intact neurologically function. This case stands out the importance of sequential TTM after repeat cardiac arrests with a return of spontaneous circulation (ROSC), demonstrating this process as a neuroprotective way of treatment.
\end{abstract}

Categories: Internal Medicine, Medical Education, Quality Improvement

Keywords: targeted temperature management, hypothermia, cardiac arrest, radiation chondronecrosis, compromised airway

\section{Introduction}

Targeted temperature management (TTM), previously known as therapeutic hypothermia (TH), has been used for at least 100 years for patients of sudden cardiac arrest (CA) [1-3]. Several studies have shown that TTM has a reasonable safety profile, and that is efficacious as a neuroprotective measure [4-5]. Targeted temperature management is commonly used for $24-72$ hours at temperatures that range between $32-36^{\circ} \mathrm{C}$ [5-6]. Sequential TTM has rarely been reported [7]. We recently had one such case.

\section{Case Presentation}

Received 06/10/2019

Review began 06/10/2019 Review ended 06/19/2019 Published 06/26/2019

๑) Copyright 2019

Casillas et al. This is an open access article distributed under the terms of the Creative Commons Attribution License CC-BY 3.0., which permits unrestricted use, distribution, and reproduction in any medium, provided the original author and source are credited.
A 59-year-old man with a history of nonmetastatic tonsillar malignancy and post radiation stridor, was referred to our service for evaluation of his upper airway. He complained of decreased cervical range of motion and severe dysphagia. An elective direct upper laryngoscopy was performed under conscious sedation. He was found to have a markedly edematous epiglottis (see Figure 1). 


\section{Cureus}

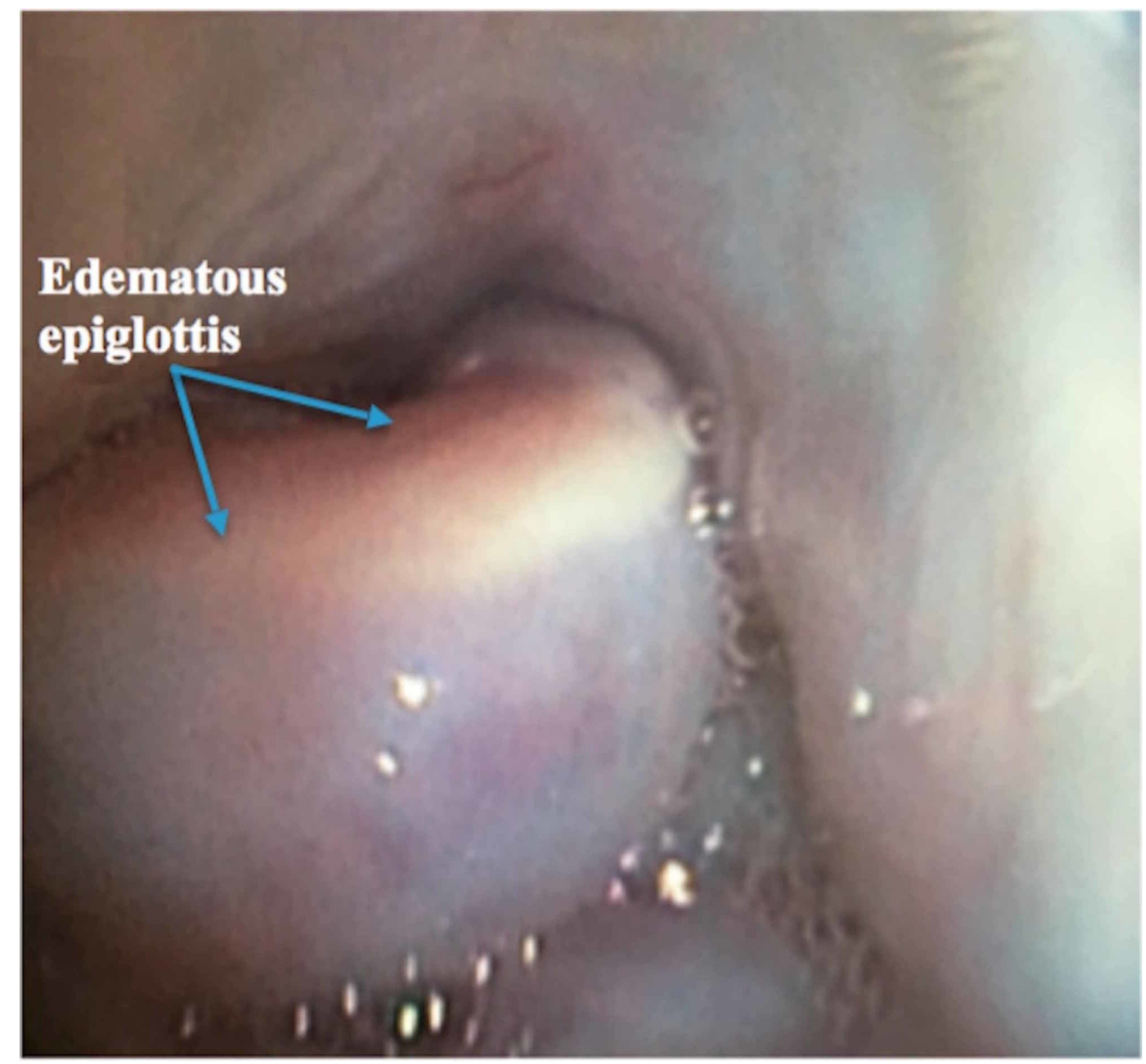

\section{FIGURE 1: Severely edematous epiglottis obstructing the view of the}

vocal cords

Within minutes, the patient's oxygen saturation dropped to $60 \%$ while breathing supplemental oxygen at $100 \%$ via a non-rebreather mask. Bag-valve ventilation was attempted with no success. His heart rate dropped to 30 beats per minute. Several attempts of intubation were unsuccessful due to the severe narrowing of his upper airway and the inability to move his neck. He developed a respiratory arrest that was followed by pulseless electrical activity. Laryngeal mask airway placement was also unsuccessful due to small oral opening. As the patient had significant fibrosis of the anterior neck, cricothyrotomy was not feasible. Cardiopulmonary resuscitation (CPR) was started and within 20 minutes, (after successful intubation was accomplished with $5 \mathrm{~F}$ endotracheal tube) return of spontaneous circulation (ROSC) was obtained. Relevant laboratory data are detailed in Table 1. 


\section{Cureus}

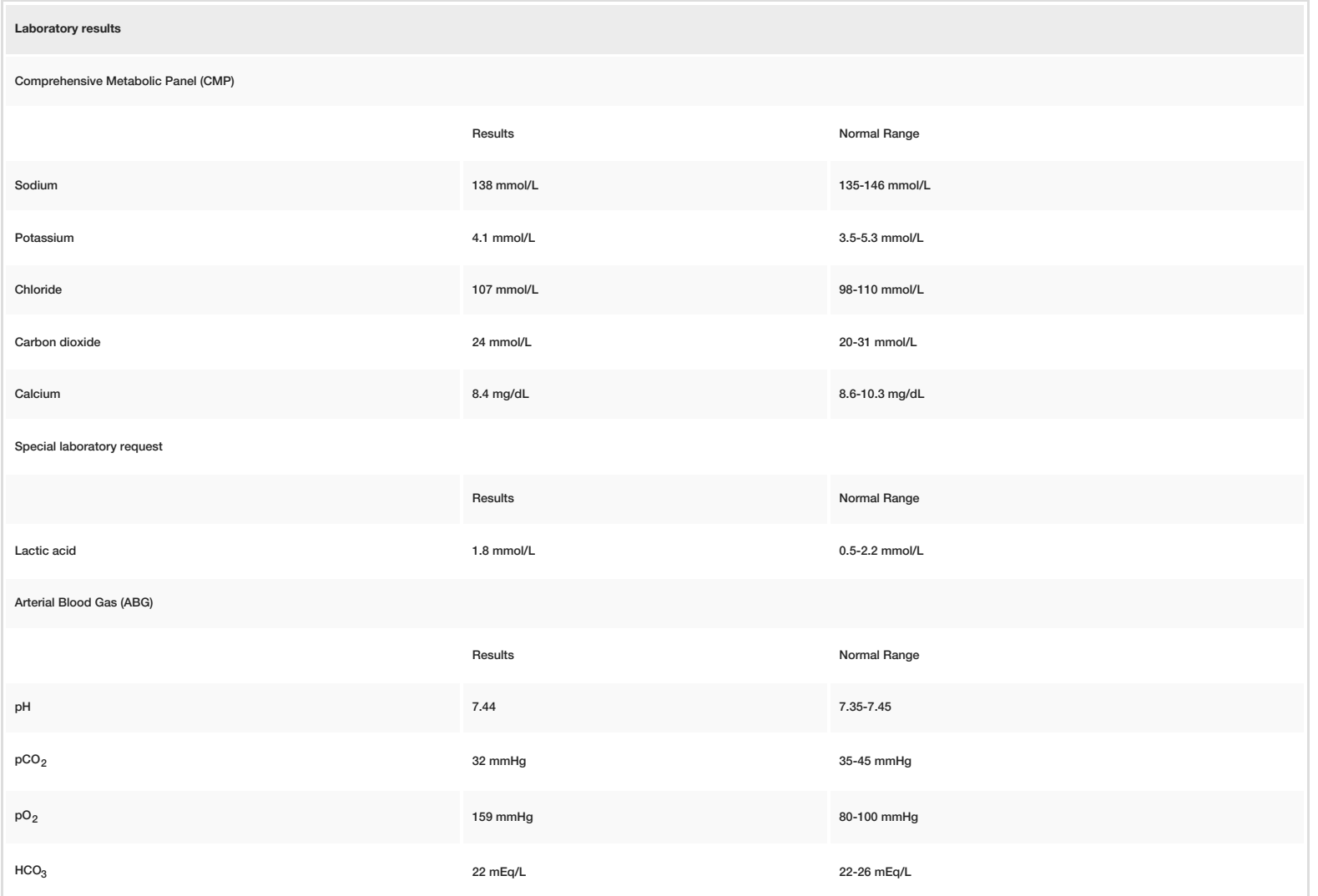

TABLE 1: Laboratory findings

The patient underwent TTM with ice packs and cooling blankets until his core temperature reached $32^{\circ} \mathrm{C}$. This temperature was maintained for 24 hours. The patient was rewarmed over 24 hours to the temperature of $36.5^{\circ} \mathrm{C}$. Mean arterial pressure (MAP), heart rate, temperature, and Glasgow coma scale (GCS) were monitored at the same time. Within minutes after discontinuation of the sedative agents, the patient began following commands, had a good gag reflex and deep tendon reflexes. The patient was extubated after having a good leak on deflation of the endotracheal tube cuff. He was able to vocalize and had no stridor. Six hours post-extubation, the patient complained of dyspnea and started having oxygen desaturation. On exam, no stridor was present and attempt of non-invasive ventilation failed.

Anesthesiology attempted intubation several times. During this process, the patient once again developed cardiopulmonary arrest. Again, CPR was initiated as per standard American Heart Association (AHA) and Advanced Cardiac Life Support (ACLS) protocol. The patient was successfully intubated again with difficulty with 5 French endotracheal tube. After 45 minutes of CPR, ROSC was achieved one more time. The patient's GCS was 3/15. At that point, a decision was made to reinitiate TTM once again. The patient was cooled to $32^{\circ} \mathrm{C}$ for 24 hours. He was then rewarmed over a 24 -hour period. However, this time, once sedative agents were weaned off, the patient remained unresponsive. His pupils were equal, round and reactive. He had no response to painful or auditory stimuli. He remained neurologically unchanged for 48 hours, and on the third day, he began opening his eyes upon auditory stimuli and withdrawing upon painful stimuli. On the fifth day post rewarming, he was fully awake and alert. He underwent permanent tracheostomy placement and gastrostomy tube placement and was weaned from assisted ventilation two days later. He was discharged to home. On three months follow up, the patient had no neurological deficits and had undergone an upper airway and esophageal reconstruction; the tracheostomy and gastrostomy were removed.

\section{Discussion}

Current practice guidelines recommend TTM in CA patients successfully resuscitated with persistent coma, as a Class I recommendation if the arrest had an initial ventricular tachycardia or ventricular fibrillation, and Class IIb recommendation from other non-shockable rhythms [8]. The European Resuscitation Council guidelines for resuscitation recommend TTM for all comatose survivors of CA regardless of initial rhythm, although the guidelines recognize a lower level of evidence for TTM in patients with a CA from nonshockable rhythms [9].

The cornerstone of management in patients that suffer a CA with ROSC is to attempt to provide the best 
possible neurological prognosis. Targeted temperature management can improve neurological recovery of these patients [10]. Although not completely clear, the alleged neuroprotective effects of TTM have several molecular and cellular mechanisms. For example, in TTM a reduction of the extracellular levels of excitatory neurotransmitters occurs [11]. Cooling also decreases the activation of the intrinsic and extrinsic apoptotic pathways, as well as, mitigates reperfusion injury, by protecting the lipoprotein membrane integrity and preventing apoptosis $[2,11]$.

Clinically, the data supports TTM in the management of these patients. One cohort study conducted by Chan et al. compared TTM and non-hypothermia-treated patients after in-hospital CA, showing a lower likelihood of survival after one time TTM (27.4\% vs $29.2 \%$ ), and a lower favorable neurological survival (17.0\% vs $20.5 \%$ [ [12]. Perman and associates also showed not only a neurological improvement but also a greater survival to hospital discharge in patients who underwent TTM than the ones who did not receive it (17.6\% vs. $28.9 \%)[13]$.

A large meta-analysis comparing survival and neurological recovery at discharge in groups that underwent TTM against groups that did not receive it, revealed that patients in TTM group were more likely to be discharged with none or minimal neurological sequelae (risk ratio 1.68 and $p$ value .006); it is worth mentioning that in this study, the patients had follow-up for six months and the TTM group had better neurological recovery (risk ratio, 1.44 and $p$ value .009) [14].

The exact duration and depth of TTM remain a matter of controversy [15-16]. To our knowledge, there have been no randomized clinical trials specifically addressing consecutive TTM in in-hospital CA. Our case depicts that consecutive TTM is safe and can be efficacious. Indeed, in our case, the patient tolerated more than one TTM "session" reaching the target temperature without any complications. We had previously described another case where a patient underwent TTM twice successfully [17]. However, the present case is different in the duration of the arrest, the pre-existing conditions, and the excellent outcome that our patient had.

\section{Conclusions}

Sequential TTM can be brain-saving and should be considered in patients that have a repeat CA with ROSC and coma. There is clearly a need to conduct a longitudinal study for sequential TTM in patients who have more than one CA.

\section{Additional Information \\ Disclosures}

Human subjects: Consent was obtained by all participants in this study. Conflicts of interest: In compliance with the ICMJE uniform disclosure form, all authors declare the following: Payment/services info: All authors have declared that no financial support was received from any organization for the submitted work. Financial relationships: All authors have declared that they have no financial relationships at present or within the previous three years with any organizations that might have an interest in the submitted work. Other relationships: All authors have declared that there are no other relationships or activities that could appear to have influenced the submitted work.

\section{Acknowledgements}

Informed consent statement: Informed written consent was obtained from the patient for publication of this report and any accompanying images. CARE checklist (2016) statement: The authors have read the CARE Checklist (2013), and the manuscript was prepared and revised according to the CARE Checklist (2016).

\section{References}

1. Alzaga AG, Cerdan M, Varon J: Therapeutic hypothermia. Resuscitation. 2006, 70:369-380. 10.1016/j.resuscitation.2006.01.017

2. Perman S, Goyal M, Neumar R, Topjian AA, Gaieski DF: Clinical applications of targeted temperature management. Chest. 2014, 145:386-393. 10.1378/chest.12-3025

3. Varon J: Therapeutic hypothermia in cardiac arrest: 206 years later! . Resuscitation. 2009, 80:1335. 10.1016/j.resuscitation.2009.08.021

4. Bernard S, Gray T, Buist M, Jones B, Silvester W, Gutteridge G, Smith K: Treatment of comatose survivors of out-of-hospital cardiac arrest with induced hypothermia. N Engl J Med. 2002, 346:557-563. 10.1056/NEJMoa003289

5. The Hypothermia after Cardiac Arrest Study Group: Mild therapeutic hypothermia to improve the neurologic outcome after cardiac arrest. N Engl J Med. 2002, 346:549-556. 10.1056/NEJMoa012689

6. Moore P: Cardiopulmonary resuscitation. In Abernathy's Surgical Secrets. Harken A, Moore E (ed): Elsevier, Philadelphia; 2018. 7:7-12. 10.1016/B978-0-323-47873-1.00002-4

7. Leong S, Chan E, Ho B, et al.: Therapeutic temperature management (TTM): post-resuscitation care for adult cardiac arrest, with recommendations from the National TTM workgroup. Singapore Med J. 2017, 58:408-410. 10.11622/smedj.2017067

8. Karcioglu O, Topacoglu H, Dikme O, Dikme O: A systematic review of safety and adverse effects in the 


\section{Cureus}

practice of therapeutic hypothermia. Am J Emerg Med. 2018, 36:1886-1894. 10.1016/j.ajem.2018.07.024 9. Scirica B: Therapeutic hypothermia after cardiac arrest. Circulation. 2013, 127:244-250. 10.1161/CIRCULATIONAHA.111.076851

10. Taccone F, Cronberg T, Friberg H, et al.: How to assess prognosis after cardiac arrest and therapeutic hypothermia. Crit Care. 2014, 18:202. 10.1186/cc13696

11. Gonzales-Ibarra G, Varon J, Lopez-Meza E: Therapeutic hypothermia: critical review of the molecular mechanisms of action. Front Neurol. 2011, 2:4. 10.3389/fneur.2011.00004

12. Chan P, Berg R, Tang Y: Association between therapeutic hypothermia and survival after in-hospital cardiac arrest. JAMA. 2016, 316:1375-1382. 10.1001/jama.2016.14380

13. Perman SM, Grossestreuer AV, Wiebe DJ, Carr BG, Abella BS, Gaieski DF: The utility of therapeutic hypothermia for post-cardiac arrest syndrome patients with an initial nonshockable rhythm. Circulation. 2015, 132:2146-2151. 10.1161/CIRCULATIONAHA.115.016317

14. Holzer M, Bernard S, Hachimi-Idrissi S, Roine RO, Sterz F, Müllner M: Hypothermia for neuroprotection after cardiac arrest: Systematic review and individual patient data meta-analysis. Crit Care Med. 2005, 33:414-418. 10.1097/01.CCM.0000153410.87750.53

15. Surani S, Varon J: The expanded use of targeted temperature management: time for reappraisal . Resuscitation. 2016, 108:A8-A9. 10.1016/j.resuscitation.2016.09.002

16. Polderman KH, Varon J: Confusion around therapeutic temperature management hypothermia after inhospital cardiac arrest?. Circulation. 2018, 137:219-221. 10.1161/CIRCULATIONAHA.117.029656

17. Gonzalez-Ibarra G, Lopez-Meza E, Castro-Rodriguez C, Varon J: Therapeutic hypothermia for the second time: is it possible?. Crit Care Shock. 2012, 15:15-16. http://criticalcareshock.org/2012/07/therapeutichypothermia-for-the-second-time-is-it-possible/. 